\title{
Caracterização do consumo alimentar, ambiente socioeconômico e estado nutricional de pré-escolares de creches municipais
}

\author{
Characteristics of dietary intake, socioeconomic \\ environment and nutritional status of \\ preschoolers at public kindergartens
}

Teresa Gontijo de CASTRO'

Juliana Farias de NOVAES ${ }^{1}$

Márcia Regina SILVA²

Neuza Maria Brunoro COSTA ${ }^{1}$

Sylvia do Carmo Castro FRANCESCHINI ${ }^{1}$

Adelson Luiz Araújo TINÔCO ${ }^{1}$

Paulo Fernando da Glória LEAL ${ }^{1}$

\section{RE S U M O}

\section{Objetivo}

Analisar o consumo alimentar, o ambiente socioeconômico, a freqüência de anemia ferropriva e o estado nutricional de pré-escolares.

\section{Métodos}

A população estudada constituiu-se de 89 crianças de 24 a 72 meses de idade, assistidas em creches municipais de Viçosa, MG. Foram avaliados: nível de hemoglobina, peso, estatura, presença de parasitose, consumo alimentar dos pré-escolares e o perfil biossocioeconômico de suas famílias.

\section{Resultados}

O estado nutricional do grupo foi considerado satisfatório, e a prevalência de anemia relativamente baixa $(11,2 \%)$. Condições adequadas de saneamento, nível razoável de escolaridade dos pais, baixo número de filhos e ausência de parasitas envolvidos com a gênese da anemia podem justificar o perfil observado. Não foi observada associação da anemia ferropriva nem com desnutrição nem com parasitose.

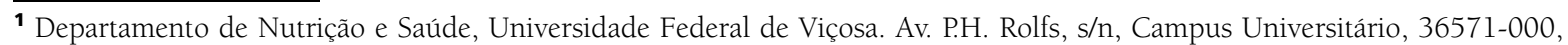

Viçosa, MG, Brasil. Correspondência para/Correspondence to: N.M.B. COSTA. E-mail: <nmbc@mail.ufv.br>.

2 Departamento de Ciência e Tecnologia de Alimentos, Universidade Federal de Viçosa. Viçosa, MG, Brasil.
} 


\section{Conclusão}

Apesar de alguns fatores biossocioeconômicos apresentarem-se favoráveis ao estado nutricional e à baixa prevalência de anemia, observa-se, entretanto, que a insuficiente renda per capita e a dieta deficiente poderão levar esse grupo de pré-escolares, no futuro, a um pior estado de saúde.

Termos de Indexação: anemia ferropriva, consumo alimentar, desnutrição infantil, estado nutricional, socioeconômico, pré-escolar.

\section{A B S T R A C T}

\section{Objective}

To evaluate the dietary intake, the socioeconomic environment, the frequency of iron deficiency anemia, and the preschoolers' nutritional status.

\section{Methods}

The evaluated population consisted of 89 children aged 24 to 72 months, who were assisted at the public nursery schools in Viçosa, MG, Brazil. Evaluation was performed for hemoglobin level, body weight, stature, parasites' presence and dietary intake of the children, as well as for their families' biological and socioeconomic profiles.

\section{Results}

The group's nutritional status was considered satisfactory, and anemia prevalence was relatively low (11.2\%). This profile may be justified by the adequate conditions of sanitation, reasonable level of parents' schooling, low numbers of children per family, and absence of parasites involved in the anemia genesis. No association was observed between iron-deficiency anemia and malnutrition, neither parasitosis.

\section{Conclusion}

Although some bio-socioeconomic factors showed favorable nutritional state and low anemia prevalence, it was observed, however, that the families' insufficient income per capita and their deficient diet will probably lead this group of children to a worse health status.

Indexing terms: anemia, iron-deficiency, dietary intake, child nutrition disorders, nutritional status, socioeconomic, child, preschool.

\section{N T R O D U Ç Ã O}

As carências nutricionais, em especial a desnutrição energético-protéica, a anemia e a deficiência de vitamina $A$ representam um dos principais problemas de saúde infantil. O crescimento tem sido reconhecido como altamente dependente de energia, proteína e micronutrientes, em especial vitamina A, zinco e ferro, e normalmente as carências desses elementos não ocorrem isoladamente' .

As evidências indicam que o meio ambiente, permeado pelas condições materiais de vida e pelo acesso aos serviços de saúde e educação, determina padrões característicos de saúde e doença na criança ${ }^{1}$. Variáveis como renda familiar, escolaridade, entre outras, estão condicionadas, em última instância, à forma de inserção das famílias no processo de produção, refletindo na aquisição de alimentos ${ }^{2}$ e, conseqüentemente, no estado nutricional.

O estado nutricional exerce influência decisiva nos riscos de morbimortalidade e no crescimento e desenvolvimento infantil, o que torna importante uma avaliação nutricional dessa população mediante procedimentos diagnósticos que possibilitem precisar a magnitude, o comportamento e os determinantes dos agravos nutricionais, assim como identificar os grupos de risco e as intervenções adequadas ${ }^{3}$.

O modelo causal da desnutrição infantil tem determinantes multicausais, com condicionantes biológicos e sociais que se relacionam com o 
atendimento (ou não) de suas necessidades básicas, como saúde, saneamento, educação e alimentação ${ }^{4}$. Quanto a essa última, os inquéritos dietéticos tornam-se instrumentos importantes para avaliação do consumo alimentar, pois permitem a identificação de deficiências de macro e micronutrientes, assim como o conhecimento dos hábitos alimentares. Ressalta-se que não existe uma metodologia de inquérito dietético ideal; porém a escolha do método adequado a um determinado propósito permite a obtenção de melhores resultados, sendo, muitas vezes, a combinação de mais de um método útil na avaliação do consumo alimentar habitual dos indivíduos 5 .

A anemia ferropriva é a carência nutricional de maior ocorrência no mundo e afeta tanto populações de países desenvolvidos quanto de países em desenvolvimento. A anemia associa-se ao retardo no desenvolvimento neuropsicomotor, comprometimento da imunidade celular e diminuição da capacidade intelectual ${ }^{6}$.

Segundo Monteiro et al. ${ }^{2}$, vários fatores podem contribuir para a anemia, tais como doenças genéticas, infecções e deficiências de diversos nutrientes. No entanto, admite-se que sua ocorrência endêmica na infância seja decorrente, principalmente, da combinação de necessidades excepcionalmente elevadas de ferro, impostas pelo crescimento, com dietas pobres no mineral, sobretudo de ferro hemínico.

Dentre as populações de risco, as crianças em idade pré-escolar constituem um grupo altamente vulnerável à deficiência de ferro, o que suscita grande preocupação na área de saúde pública em razão dos prejuízos que acarreta ao desenvolvimento dessas crianças?.

Este estudo analisa o consumo alimentar, o ambiente socioeconômico e o estado nutricional de pré-escolares em creches municipais de Viçosa, MG.

\section{MÉ T O D O S}

O município de Viçosa, MG, conta com doze creches municipais que atendem 250 crianças. Este estudo, descritivo, foi realizado com 87 crianças de 24 a 72 meses (compreendendo $34,8 \%$ do total de crianças atendidas nas creches municipais), pertencentes a cinco creches, selecionadas aleatoriamente. A variação do tamanho amostral nas diferentes variáveis deve-se à ausência de algumas crianças nas creches durante os dias agendados para realização da coleta de dados.

Foi aplicado um questionário de freqüência de consumo alimentar às mães ou aos responsáveis pelas crianças a fim de avaliar o consumo de nove grupos de alimentos, quais sejam, cereais e massas, leguminosas, frutas, ovos, hortaliças, raízes e tubérculos, carnes, doces e gorduras e leite e derivados. As freqüências de consumo foram classificadas como consumo raro, de uma a três vezes por semana, e de quatro a sete vezes por semana. Como consumo raro foram considerados os consumos quinzenal, mensal, ocasional, ou a não-ingestão8 .

Juntamente com o questionário de freqüência alimentar, foi avaliada a dieta por meio de pesagem direta dos alimentos consumidos individualmente pelas crianças nas creches (colação, almoço e lanche) ${ }^{8}$, utilizando-se uma balança digital com capacidade de dois quilos e sensibilidade de $1 \mathrm{~g}$. As refeições realizadas no domicílio (desjejum e jantar) foram avaliadas pelo método recordatório 24 horas, tendo as mães como respondentes. Esses dois registros foram realizados durante três dias úteis, sendo os dados analisados em conjunto, de modo que um inquérito alimentar complementava o outro com os tipos de refeições realizadas durante um dia pelas crianças a fim de se conhecer a ingestão habitual de energia e nutrientes. Os nutrientes analisados foram proteínas, ferro, cálcio, vitamina A e vitamina $C$. A prevalência de inadequação para proteínas, ferro, vitamina $C$ e vitamina $A$ foi calculada de acordo com a Necessidade Média Estimada (Estimated Average Requirement, EAR) do National Research Council ${ }^{9-12}$. A ingestão de cálcio foi analisada com base nos valores da Ingestão Adequada (Adequate Intake, Al), já que 
não existe EAR para esse nutriente ${ }^{13}$. A avaliação da ingestão de energia foi feita utilizando a Necessidade Energética Estimada (Estimated Energy Requirement, EER), ou seja, o consumo de energia necessário para atender o balanço energético compatível com um bom estado de saúde $^{10}$. Os cálculos das dietas e suas análises foram feitos com auxílio do software Diet-Pro ${ }^{14}$, sendo a ingestão mediana e o percentual de inadequação de energia e nutrientes analisados pelo software Epi Info ${ }^{16}$. Foi calculado o consumo mediano de ingestão e não o consumo médio, uma vez que o desvio-padrão apresenta grande variabilidade, comum em estudos dietéticos. De fato, a ingestão de nutrientes raramente apresenta distribuição normal.

É importante ressaltar que a escolha do tipo de inquérito dietético irá depender das características dos indivíduos da amostra e do objetivo do estudo. Neste caso, a utilização dos três inquéritos alimentares citados neste trabalho está pertinente com as características de sua amostra, assim como com o objetivo proposto pelo estudo.

Para avaliar o perfil biossocioeconômico das famílias, adotou-se a metodologia de entrevista com as mães ou com os responsáveis pelas crianças, por meio da aplicação de um questionário semi-estruturado. Foram avaliadas 79 famílias, abrangendo a totalidade das 87 crianças que compõem a amostra. As variáveis analisadas foram renda familiar e per capita, escolaridade dos pais, número de filhos, densidade familiar, número de dormitórios nos domicílios, condições de saneamento ambiental, condições de nascimento (peso da criança ao nascer, tipo de parto, idade gestacional) e demanda por serviços de saúde.

Foram verificadas as medidas antropométricas de peso e altura de acordo com as técnicas propostas por Jelliffe ${ }^{15}$. Para medida de peso, utilizou-se balança portátil, digital, eletrônica, com capacidade de $150 \mathrm{~kg}$ e sensibilidade de $50 \mathrm{~g}$, sendo a aferição da altura feita por meio de estadiômetro com extensão de dois metros, dividido em centímetros e subdividido em milímetros. A análise dos índices peso/idade (P/I), peso/estatura (P/E) e estatura/idade (E/I) foi feita pelo critério escore-Z com auxílio do software Epi Info ${ }^{16}$, sendo os índices avaliados com base na referência do National Center for Health Statistics (NCHS) ${ }^{17}$. Foram consideradas desnutridas as crianças cujos índices encontravam-se abaixo de $-2 z$, e obesas aquelas cujos índices P/l e P/E estavam acima de $+2 z$. As crianças cujos índices se encontravam entre $-1 z$ e -2z foram classificadas como em risco nutricional para desnutrição, e aquelas no intervalo de $+1 z$ e $+2 z$, em risco nutricional para obesidade ${ }^{17}$.

Amostras de sangue foram coletadas, por punção venosa, para determinação da concentração de hemoglobina, medida automaticamente em aparelho Cobas Argos-Roche. Foram consideradas anêmicas as crianças que apresentaram concentração de hemoglobina inferior a $11 \mathrm{~g} / \mathrm{dL}^{18}$. O grupo foi submetido ao exame parasitológico de fezes. As anêmicas e parasitadas foram tratadas e acompanhadas pelo pediatra da Secretaria Municipal de Saúde, já que este não é um programa, e sim uma parceria com o Departamento de Nutrição e Saúde da Universidade Federal de Viçosa e com a Secretaria Municipal de Saúde, que ocorre freqüentemente em estudos realizados neste município.

Foi aplicado o teste do qui-quadrado para avaliar a associação entre anemia ferropriva e estado nutricional e anemia ferropriva e parasitose intestinal, utilizando-se nível de significância de $5 \%(p<0,05)$.

\section{RES ULTA D OS}

Do total das crianças, $61,0 \%$ pertenciam ao gênero masculino e 39,0\% ao feminino. Observou-se que, com exceção da proteína, todos os demais nutrientes, vitaminas e minerais encontravam-se abaixo da necessidade média estimada (EAR) para a faixa etária em estudo. Verificou-se que 75,7\% das crianças apresentaram inadequação da ingestão energética em relação à necessidade energética estimada (EER). Apesar 
de $92,8 \%$ dos pré-escolares apresentarem ingestão de cálcio abaixo do valor da ingestão adequada (Al), não é possível calcular a prevalência de inadequação para os nutrientes cuja EAR não está disponível (Tabela 1).

Tabela 1. Consumo mediano e freqüência de inadequação de energia, proteínas, vitaminas e minerais dos pré-escolares $(n=87)$ de creches municipais de Viçosa, MG, 1999.

\begin{tabular}{|c|c|c|}
\hline Energia e nutrientes & Mediana & Inadequação (\%) \\
\hline Energia $(\mathrm{kcal})^{*}$ & 1221,2 & 75,7 \\
\hline Proteínas $(\mathrm{g})^{\star *}$ & 41,0 & 0,0 \\
\hline Vitamina C $(\mathrm{mg})^{* *}$ & 13,1 & 72,8 \\
\hline Ferro $(\mathrm{mg})^{* *}$ & 4,5 & 21,4 \\
\hline Vitamina $A(\mu g R E)^{* *}$ & 336,9 & 22,8 \\
\hline Cálcio (mg) & 285,0 & - \\
\hline
\end{tabular}

${ }^{*} \mathrm{EER}=$ necessidade energética estimada; ${ }^{* *} \mathrm{EAR}=$ necessidade média estimada.

As freqüências de consumo dos grupos alimentares pelas crianças, sendo cada grupo representado pelo alimento mais consumido na freqüência de quatro a sete vezes por semana, foram margarina (doces e gorduras), banana (frutas), batata (raízes e tubérculos), frango (carnes), alface (hortaliças), feijão (leguminosas), leite (leite e derivados) e arroz (cereais e massas) (Tabela 2). Os resultados permitem verificar que as maiores freqüências de consumo estavam nos grupos dos cereais e massas, leguminosas, leite e derivados, e doces e gorduras. Observou-se baixa freqüência no consumo de frutas, hortaliças e raízes e tubérculos.

Também o consumo de carnes foi pouco freqüente, visto que se constatou $25,7 \%$ dos pré-escolares consumindo carne raramente, $53,8 \%$ de uma a três vezes por semana, e apenas $20,5 \%$ de quatro a sete vezes por semana.

É importante ressaltar que os dados dos três inquéritos dietéticos não foram apresentados juntos, uma vez que a Pesagem Direta e o Recordatório 24 horas são métodos quantitativos de avaliação do consumo alimentar, e a Freqüência de Alimentos é um método qualitativo.

$\mathrm{Na}$ avaliação do perfil biossocioeconômico das famílias (Tabela 3), verificou-se que a quase totalidade das famílias $(93,3 \%)$ tinha rendimento inferior a cinco salários mínimos, e, 60,0\% delas, rendimento per capita inferior a meio salário mínimo.

Quanto à escolaridade, 44,7\% das mães possuíam nível menor ou igual a quatro anos de estudo e $55,3 \%$ mais de quatro anos de ensino formal. Com referência à escolaridade paterna, $42,6 \%$ tinham escolaridade menor ou igual a quatro anos e $57,4 \%$ maior que quatro anos de ensino formal.

Tabela 2. Freqüência de consumo dos diferentes grupos alimentares por pré-escolares $(n=79)$ de creches municipais de Viçosa, $M G$, 1999

\begin{tabular}{lccc}
\hline \multirow{2}{*}{ Grupo alimentar } & \multicolumn{3}{c}{ Freqüência de consumo (\%) } \\
\cline { 2 - 4 } & Raramente & 1 a 3 vezes/semana & 4 a 7 vezes/semana \\
\hline Cereais e massas & 10,25 & 1,25 & 88,50 \\
Leguminosas & 5,10 & 1,25 & 93,65 \\
Frutas & 21,80 & 53,80 & 24,40 \\
Ovos & 15,40 & 66,60 & 18,00 \\
Hortaliças & 32,00 & 51,30 & 16,70 \\
Raízes e tubérculos & 32,00 & 51,30 & 16,70 \\
Carnes & 25,70 & 53,80 & 20,50 \\
Leite e derivados & 9,00 & 5,20 & 85,80 \\
Doces e gorduras & 27,00 & 23,00 & 50,00 \\
\hline
\end{tabular}


Tabela 3. Caracterização dos domicílios e das famílias dos pré-escolares ( $n=79)$ de creches municipais de Viçosa, MG, 1999.

\begin{tabular}{|c|c|c|c|}
\hline \multirow{2}{*}{ Variáveis } & \multirow{2}{*}{$\mathrm{n}$} & \multicolumn{2}{|c|}{ Freqüência } \\
\hline & & $\%$ & Acumulada (\%) \\
\hline \multicolumn{4}{|l|}{ Renda familiar $^{*}$} \\
\hline$<1 S M$ & 6 & 8,0 & 8,0 \\
\hline $1-3 \mathrm{sm}$ & 52 & 69,3 & 77,3 \\
\hline $31-5 s m$ & 12 & 16,0 & 93,3 \\
\hline $5 \mid-10 s m$ & 5 & 6,7 & 100,0 \\
\hline \multicolumn{4}{|l|}{ Renda per capita* } \\
\hline$<0,5 \mathrm{sm}$ & 45 & 60,0 & 60,0 \\
\hline $0,5 \mid-1 \mathrm{sm}$ & 24 & 32,0 & 92,0 \\
\hline$>1 \mathrm{sm}$ & 6 & 8,0 & 100,0 \\
\hline \multicolumn{4}{|l|}{ Escolaridade da mãe } \\
\hline$\leq 4$ anos de ensino formal & 34 & 44,7 & 44,7 \\
\hline$>4$ anos de ensino formal & 42 & 55,3 & 100,0 \\
\hline \multicolumn{4}{|l|}{ Escolaridade do pai ${ }^{*}$} \\
\hline$\leq 4$ anos de ensino formal & 29 & 42,6 & 42,6 \\
\hline$>4$ anos de ensino formal & 39 & 57,4 & 100,0 \\
\hline \multicolumn{4}{|l|}{ Número de dormitórios } \\
\hline 1 & 20 & 25,3 & 25,3 \\
\hline 2 & 27 & 34,2 & 59,5 \\
\hline 3 & 22 & 27,8 & 87,3 \\
\hline 4 & 8 & 10,2 & 97,5 \\
\hline 5 & 2 & 2,5 & 100,0 \\
\hline \multicolumn{4}{|c|}{ Número de pessoas por domicílio } \\
\hline $1-4$ pessoas & 35 & 44,4 & 44,4 \\
\hline $5-8$ pessoas & 39 & 49,4 & 93,8 \\
\hline$>9$ pessoas & 5 & 6,2 & 100,0 \\
\hline \multicolumn{4}{|l|}{ Número de filhos* } \\
\hline $1-\mid 2$ & 38 & 48,7 & 48,7 \\
\hline $2-14$ & 37 & 47,4 & 96,1 \\
\hline $4-6$ & 3 & 3,9 & 100,0 \\
\hline$>6$ & 0 & 0,0 & 0 \\
\hline \multicolumn{4}{|l|}{ Água tratada } \\
\hline Sim & 78 & 98,7 & 98,7 \\
\hline Não & 1 & 1,3 & 100,0 \\
\hline \multicolumn{4}{|l|}{ Serviço de esgoto } \\
\hline $\operatorname{Sim}$ & 79 & 100,0 & 100,0 \\
\hline Não & 0 & 0 & 0 \\
\hline
\end{tabular}

sm= Salário mínimo em dezembro de 1999; Sem informação= 4 (renda familiar), 4 (renda per- capita), 3 (escolaridade materna), 11 (escolaridade paterna), 1 (número de filhos).

Na maioria dos domicílios havia de um a dois dormitórios $(59,5 \%)$, e grande parte das famílias constituía-se de cinco a oito membros $(49,4 \%)$. A quase totalidade das famílias $(96,1 \%)$ tinha até quatro filhos, e quase metade delas $(48,7 \%)$, até dois filhos.

Quanto às condições sanitárias das residências, verificou-se que $98,7 \%$ dispunham de água tratada, tendo como origem água de rede pública, enquanto $100,0 \%$ estavam conectadas à rede de esgoto.

Em relação às condições de nascimento (Tabela 4), segundo relato das mães, 10,3\% das crianças haviam nascido com baixo peso $(<2500 \mathrm{~g})$, $36,8 \%$ com peso insuficiente ( 2500 a 2 999g) e apenas $52,9 \%$ com peso adequado (3000 a 
4 000g). A maioria das crianças $(94,7 \%)$ nasceu com idade gestacional adequada (a termo). Quanto ao tipo de parto, 26,3\% nasceram de parto normal e 73,7\% de cesariana.

Na demanda de serviços de saúde (Tabela 4), os setores públicos foram os mais procurados (posto de saúde municipal e hospitais), sendo a procura por serviços privados relatada em apenas $6,5 \%$ das famílias.

A classificação do estado nutricional dos pré-escolares, segundo o critério escore-Z $Z$ para os índices $\mathrm{P} / \mathrm{I}, \mathrm{P} / \mathrm{E}$ e E/l, para os quais foram encontrados valores de $59,8 \%, 70,2 \%$ e $63,3 \%$ de eutrofia, respectivamente. Não se observou desnutrição aguda nesse grupo, mas apenas crônica ou pregressa caracterizada pela baixa estatura em 3,5\% das crianças, que foi inferior à obesidade (5,7\% para o índice P/l e 4,6 \% para o índice P/E). No entanto, $27,6 \%, 19,5 \%$ e $14,9 \%$ das crianças encontravam-se em risco para desnutrição, considerando-se os índices P/I, P/E e E/I, respectivamente. Já para obesidade, 6,9\% e $5,7 \%$ apresentavam-se em risco, com índices $\mathrm{P} / \mathrm{I}$ e P/E situados entre +1 e +2 escore-Z, respectivamente (Tabela 5). Não foi encontrada associação entre anemia ferropriva e desnutrição $(p>0,05)$.

Tabela 4. Características do nascimento dos pré-escolares $(n=79)$ de creches municipais de Viçosa, MG, 1999.

\begin{tabular}{|c|c|c|c|}
\hline \multirow{2}{*}{ Variáveis } & \multirow{2}{*}{$n$} & \multicolumn{2}{|c|}{ Freqüência } \\
\hline & & $\%$ & Acumulada (\%) \\
\hline \multicolumn{4}{|l|}{ Peso ao nascer ${ }^{*}$} \\
\hline $3000-4000 \mathrm{~g}$ & 36 & 52,9 & 52,9 \\
\hline $2500-2999 \mathrm{~g}$ & 25 & 36,8 & 89,7 \\
\hline$<2500 \mathrm{~g}$ & 7 & 10,3 & 100,0 \\
\hline \multicolumn{4}{|c|}{ Condições do nascimento* } \\
\hline A termo & 72 & 94,7 & 94,7 \\
\hline Pré-termo & 4 & 5,3 & 100,0 \\
\hline \multicolumn{4}{|l|}{ Tipo de parto* } \\
\hline Normal & 20 & 26,3 & 26,3 \\
\hline Cesário & 56 & 73,7 & 100,0 \\
\hline \multicolumn{4}{|c|}{ Acesso aos serviços de saúde ${ }^{*}$} \\
\hline \multicolumn{4}{|l|}{ Posto de Saúde* } \\
\hline Sim & 51 & 66,2 & 66,2 \\
\hline Não & 26 & 33,8 & 100,0 \\
\hline \multicolumn{4}{|l|}{ Hospital $^{*}$} \\
\hline Sim & 25 & 32,5 & 32,5 \\
\hline Não & 52 & 67,5 & 100,0 \\
\hline \multicolumn{4}{|c|}{ Consultórios particulares ${ }^{*}$} \\
\hline Sim & 5 & 6,5 & 6,5 \\
\hline Não & 72 & 93,5 & 100,0 \\
\hline
\end{tabular}

* Sem informações= 11 (peso ao nascer), 3 (condições de nascimento), 3 (tipo de parto), 2 (acesso aos serviços de saúde).

Tabela 5. Distribuição percentual dos índices P/I, P/E e E/I quanto aos intervalos de escore-Z, dos pré-escolares ( $n=87)$ de creches municipais de Viçosa, MG, 1999.

\begin{tabular}{lccccc}
\hline Índices & $<-2 \mathrm{z}(\%)$ & $-2 \mathrm{z} a-1 \mathrm{z}(\%)$ & $-1 \mathrm{z} a+1 \mathrm{z}(\%)$ & $+1 \mathrm{z} \mathrm{a}+2 \mathrm{z}(\%)$ & $>+2 \mathrm{z}(\%)$ \\
\hline $\mathrm{P} / \mathrm{I}$ & 0,0 & 27,6 & 59,8 & 6,9 & 5,7 \\
$\mathrm{P} / \mathrm{E}$ & 0,0 & 19,5 & 70,2 & 5,7 & 4,6 \\
$\mathrm{E} / \mathrm{I}$ & 3,5 & 14,9 & 63,3 & 17,2 & 1,1 \\
\hline
\end{tabular}

$\mathrm{P} / \mathrm{I}=$ peso/idade; $\mathrm{P} / \mathrm{E}=$ peso/estatura; $\mathrm{E} / \mathrm{I}=$ estatura/idade; $\mathrm{Z}=$ escore- $\mathrm{Z}$. 
A anemia ferropriva $(\mathrm{Hb}<1 \mathrm{~g} / \mathrm{dL})$ foi detectada em $11,2 \%$ do total dos pré-escolares, e a parasitose em $36,7 \%$. Os parasitas de ocorrência mais comum no grupo em estudo foram Entamoeba histolytica, Escherichia coli, Giardia lamblia, Endolimax nana e Ascaris lumbricoides. Não foi verificada a associação de anemia ferropriva com parasitose $(p>0,05)$.

\section{I S C U S S Ã O}

O déficit energético verificado $(75,7 \%)$ pode ser devido ao pequeno porcionamento dos alimentos (cereais, leguminosas, raízes e tubérculos) na dieta das crianças nos dias em que foram realizados os inquéritos dietéticos, o mesmo ocorrendo para o grupo do leite e derivados (mais significativamente representado pelo leite), cuja ingestão freqüente, relatada no inquérito de freqüência alimentar, contrasta com a baixa mediana de ingestão de cálcio na dieta dos préescolares.

Em pesquisa realizada em Salvador, observou-se acentuada tendência para inadequação de energia, que atingia $20,9 \%$ das crianças com 48 a 60 meses. Além disso, o consumo médio per capita de produtos lácteos por essa faixa etária estava abaixo dos $500 \mathrm{~mL}$ diários preconizados ${ }^{1}$

Apenas pequeno percentual das crianças $(20,5 \%)$ relatou alta freqüência no consumo de carnes (de quatro a sete vezes por semana), o que, provavelmente, explica a inadequação de ferro na dieta das crianças $(21,4 \%)$. Outro fator que, possivelmente, tenha contribuído para a baixa adequação do ferro dietético foi o baixo consumo per capita de carne, observado nas refeições realizadas nas creches.

Resultados semelhantes foram observados em estudo realizado com crianças de zero a 59 meses, em São Paulo, onde se constatou que, em todas as idades, o aporte dietético de ferro estava abaixo das quantidades recomendadas para o consumo ${ }^{19}$.
Além desses fatores, é possível que a baixa freqüência no consumo de frutas e hortaliças estivesse agravando a deficiência de ferro encontrada nas crianças, pois a esses grupos pertencem os alimentos fontes de vitaminas C e A. A importância dessas vitaminas na biodisponibilidade do ferro dietético não-hemínico é conhecida, uma vez que elas agem no aumento de sua absorção ${ }^{20}$. Vale ressaltar que a inadequação de vitamina $\mathrm{C}$ e $\mathrm{A}$ obtida pelos inquéritos dietéticos foi de $72,8 \%$ e $22,8 \%$, respectivamente.

Cerca de 77,3\% das famílias apresentavam renda total inferior a três salários mínimos, e $60,0 \%$ dessas tinham renda inferior a meio salário mínimo per capita, ou seja, grande parte das famílias vivia abaixo do que se convencionou chamar "linha de pobreza". Segundo Monteiro et al. ${ }^{21}$, a importância do nível de renda na determinação de condições de saúde decorre da ampla influência que esta exerce na possibilidade de aquisição e utilização de bens e serviços essenciais à manutenção do estado de saúde, tais como alimentação, moradia, vestuário e saneamento.

Mais da metade das mães (55,3\%) e dos pais $(57,4 \%)$ possuía nível de escolaridade maior que quatro anos de ensino formal, o que poderia estar contribuindo para o melhor estado nutricional e para a baixa freqüência de anemia observados nessas crianças quando comparadas a outras, em estudos semelhantes ${ }^{22,4}$. O saber ler e escrever da mãe influencia, favoravelmente, o estado de nutrição do filho, pois o meio informal de transferência de conhecimentos da mãe pode se articular com outros fatores do meio social, como trabalho, renda e condições de ambiente físico ${ }^{4}$.

Quanto à densidade familiar, quase metade dos domicílios $(44,4 \%)$ tinha de uma a quatro pessoas, o que indica uma diminuição no tamanho das famílias caracterizadas como numerosas. O número de filhos também era reduzido, sendo observado que $96,1 \%$ das famílias tinham de um a quatro filhos. Essa redução no número de filhos também foi constatada pela Pesquisa Nacional sobre Demografia e Saúde ${ }^{23}$, cujos resultados 
mostram que a média geral foi 1,9 filho nascido vivo para todas as mulheres.

As condições sanitárias dos domicílios eram satisfatórias, uma vez que $98,7 \%$ das residências tinham acesso à água tratada e 100,0\% à coleta de esgoto. Os distúrbios do estado de saúde e nutricional, durante os primeiros anos de vida, são determinados por diversos fatores. Nos países em desenvolvimento, esses distúrbios estão associados às condições gerais de vida e ao nível de atendimento das necessidades básicas da população²2

A freqüência de baixo peso $(10,3 \%)$ ao nascer foi alta, quando comparada a alguns indicadores nacionais. Segundo dados do Ministério da Saúde ${ }^{24}$, a incidência de baixo peso ao nascer no país foi de 7,9\% em 1998, e de $8,1 \%$ no município de Viçosa em 1999. Em estudo realizado no sul do Brasil, constatou-se que crianças nascidas com baixo peso estavam expostas a um risco onze vezes maior de morte no primeiro ano de vida e cinco vezes maior de vir a apresentar deficiência ponderal no segundo ano de vida ${ }^{25}$.

O déficit estatural das crianças estudadas $(3,5 \%)$ foi inferior ao encontrado em pré-escolares $(12,5 \%)$ assistidos em creches das diferentes regiões do Brasil|26. O comprometimento da altura foi também observado em $22,5 \%$ das crianças de zero a sete anos, atendidas em período integral pelos CEC municipais (centros educacionais e creches), em Piracicaba, SP22. No entanto, o risco nutricional para desnutrição $(27,6 \%$ para $P / I$, $19,5 \%$ para P/E e 14,9\% para E/I) foi substancialmente maior que o esperado na distribuição normal, na qual $13,6 \%$ de indivíduos estão situados entre -2 e -1 desvio-padrão.

A prevalência de anemia encontrada nos pré-escolares das cinco creches municipais $(11,2 \%)$ foi menor do que a observada em outros estudos realizados no país. Estudo avaliando as condições de vida, saúde e nutrição em Salvador detectou um percentual de $46,4 \%$ de anemia entre crianças menores de cinco anos ${ }^{1}$. A mesma prevalência de anemia $(46,9 \%)$ foi encontrada por Monteiro et al. ${ }^{2}$ no município de São Paulo.
Não foi encontrada associação entre anemia e parasitose, provavelmente devido à baixa ocorrência de ancilostomose nas crianças, parasita mais envolvido na etiopatogenia da anemia ferropriva. Observações semelhantes foram encontradas por Monteiro \& Szarfarc ${ }^{27}$ em crianças menores de cinco anos de idade, em estudo sobre as condições de saúde dessas, no município de São Paulo.

\section{CONCLUSÃO}

Este estudo evidenciou que as crianças assistidas nas creches municipais de Viçosa, MG, apresentavam estado nutricional satisfatório e baixa prevalência de anemia, quando comparadas às crianças de outros municípios. Os fatores que, possivelmente, contribuíram para essa melhor situação de saúde foram: nível razoável de escolaridade dos pais, número pequeno de filhos, condições adequadas de saneamento dos domicílios onde viviam os pré-escolares e baixa freqüência de parasitas envolvidos na gênese da anemia ferropriva. Além disso, a baixa prevalência de anemia nas crianças pode ser devida à proteção que, teoricamente, o serviço institucionalizado (creche) deve proporcionar. No entanto, a baixa renda per capita da maioria das famílias e a dieta deficiente dessas crianças podem, futuramente, vir a deteriorar a situação de saúde dos pré-escolares.

\section{A GRADECIMENTOS}

Os autores agradecem ao CNPq pelo suporte financeiro, ao Programa Especial de Treinamento (PET/NUT/UFV), à Secretaria Municipal de Educação, aos pais, às crianças estudadas, aos funcionários das creches e aos alunos de Nutrição da Universidade Federal de Viçosa pelo apoio.

\section{REFER Ê N CIAS}

1. Assis AMO, Barreto ML. Condições de vida, saúde e nutrição na infância em Salvador. Salvador: UFBA; 2000. 
2. Monteiro CA, Szarfarc SC, Mondini L. Tendência secular da anemia na infância na cidade de São Paulo (1984-1996). Rev Saúde Pública. 2000; 34(6):62-72.

3. Ribas DLB, Philippi ST, Tanaka ACD'A, Zorzatto JR. Saúde e estado nutricional infantil de uma população da região centro-oeste do Brasil. Rev Saúde Pública. 1999; 33(4):358-65.

4. Engstrom EM, Anjos LA. Déficit estatural nas crianças brasileiras: relação com condições sócio-ambientais e estado nutricional materno. Cad Saúde Pública. 1999;15(3):559-67.

5. Duarte AC, Castellani FR. Semiologia Nutricional. Rio de Janeiro: Axcel Books; 2002.

6. Neuman NA, Tanaka OY, Szarfarc SC, Guimarães PRV, Victora CG. Prevalência e fatores de risco para anemia no sul do Brasil. Rev Saúde Pública.2000; 34(1):56-63.

7. Schimitz BAS, Picanço MR, Aquino KKNC, Bastos J, Giorgini E, Cardoso R, et al. Prevalência de desnutrição e anemia em pré-escolares de Brasília-Brasil. Pediatr Mod. 1998; 34 (4):155-64.

8. Cintra IP, Von Der Heyde HED, Schmitz BA, Franceschini SCC, Taddei JA, Sigulem DM. Métodos de Inquéritos Dietéticos. Cad Nutrição. 1997; 13:11-23.

9. Institute of Medicine. National Research Council. Dietary References Intakes: Applications in Dietary Assessment. Washington (DC): National Academy Press; 2001.

10. Institute of Medicine. National Research Council. Dietary Reference Intakes for Energy, Carbohydrate, Fiber, Fat, Fatty Acids, Cholesterol, Protein, and Amino Acids (Macronutrients). Washington (DC): National Academy Press; 2002.

11. Institute of Medicine. National Research Council. Dietary Reference Intakes for Vitamin A, Vitamin K, Arsenic, Boron, Chromium, Copper, lodine, Iron, Manganese, Molybdenum, Nickel, Silicon, Vanadium, and Zinc. Washington (DC): National Academy Press; 2002.

12. Institute of Medicine. National Research Council. Dietary Reference Intakes for Vitamin C, Vitamin E, Selenium, and Carotenoids. Washington (DC): National Academy Press; 2002.

13. Institute of Medicine. National Research Council. Dietary Reference Intakes for Calcium, Phosphorus, Magnesium, Vitamin D, and Fluoride. Washington (DC): National Academy Press; 1999.

14. Monteiro JBR, Esteves E. Diet Pro: sistema de suporte à avaliação nutricional e prescrição de dietas. Versão 3.0. Viçosa: Agromídia; 2000.
15. Jelliffe DB. Evaluación del estado de nutrición de la comunidad. Ginebra: Organización Mundial e Salud; 1968. OMS - Série de monografias.

16. Dean AG, et al. Epi Info [computer program]. Version 6: a word processing, database, and statistics program for epidemiology on micro-computers. Atlanta, Georgia: Centers of Disease Control and Prevention; 1994.

17. World Health Organization. Physical status: the use and interpretation of antropometry. Geneva: WHO; 1995. Technical Report Series, 854.

18. World Health Organization. Iron Deficiency Anaemia. Assessment prevention and control. A guide for programme managers. Geneva: WHO; 2001.

19. Szarfarc SC, Monteiro CA, Meyer M, Tudisco ES, Reis IM. Estudo das condições de saúde das crianças do município de São Paulo, SP, 1984/1985. Rev Saúde Pública. 1988; 22(4):266-72.

20.Garcia-Casal MN, Layrisse M, Solano L, Barón A. Vitamin $A$ and $\beta$-carotene can improve nonheme iron absorption from rice, wheat and corn by humans. J Nutr. 1998;128(3):646-50.

21. Monteiro CA, Zuñiga HPP, Benício MHD'A, Szarfarc SC. Estudo das condições de saúde das crianças do município de São Paulo, SP. Rev Saúde Pública. 1986; 20(6):435-45.

22. Silva MV, Sturion GL. Frequência à creche e outros condicionantes do estado nutricional infantil. Rev Nutr. 1998; 11(1):58-68.

23. Pesquisa Nacional sobre Demografia e Saúde, 1996. Rio de Janeiro: Sociedade Civil Bem-Estar Familiar no Brasil; 1996.

24. Data Sus. Ministério da Saúde [Internet]. Viçosa: Ministério da Saúde. [acesso 2001 jul 20]. Disponível em: http://www.saude.gov.br

25. Victora CG, Barros FC, Vaughan JP, Teixeira AM. Birthweight and infant mortality: a longitudinal study of 5914 Brazilian children. Int J Epidemiol. 1987; 16(2):239-45.

26. Silva $M$, Ometto $A M H$, Furtuoso COM, Pipitone MAP, Sturion GL. Acesso à creche e estado nutricional das crianças brasileiras: diferenças regionais, por faixa etária e classes de renda. Rev Nutr. 2000; 13(3):193-99.

27. Monteiro CA, Szarfarc SC. Estudo das condições de saúde das crianças no município de São Paulo, SP (Brasil), 1984-1985. Rev Saúde Pública. 1987; 21(3):225-60.

Recebido para publicação em 3 de setembro de 2001 e aceito em 3 de agosto de 2004. 